\title{
Sinderes
}

\section{La formación de habilidades investigativas en los futuros profesores de educación inicial}

\author{
The training of investigative skills in the \\ future teachers of initial education
}

\section{Autores \\ María Eugenia Soto Muñoz* \\ Recibido: 25 de septiembre de 2017 . Aprobado: 3 de noviembre de 2017.}

* Educador de Párvulos.

Académico Departamento de Didáctica.

Facultad de Educación. Universidad Católica de la

Santísima Concepción. Chile

mesoto@ucsc.cl

\section{Resumen}

El artículo presenta reflexiones surgidas luego de realizar una pasantía financiada por el Ministerio de Educación Chileno a una Institución Universitaria en Medellín, que tuvo como propósito conocer experiencias en la formación inicial de docentes en Colombia, de tal forma de contribuir con experiencia y conocimientos a la formación de futuros docentes de la Facultad de Educación de una universidad regional del Consejo de Rectores de Universidades Chilenas. La pasantía permitió establecer diferencias en la Formación Inicial de Educadoras de Párvulos, destacando en el análisis la discrepancia que existe en cuanto a la formación de habilidades investigativas en estos estudiantes. El análisis reflexivo permite argumentar respecto a la necesidad de formar habilidades investigativas en los futuros profesores para permitir el desarrollo de la investigación pedagógica en Chile, y así resolver problemas tendientes a mejorar la calidad de la educación en todos sus niveles. 
Palabras clave: Investigación of Rectors of Chilean Universities. pedagógica, habilidades investigativas, The internship allowed establishing formación de docentes.

\author{
Abstract \\ The article presents reflections arisen \\ after an internship funded by the Chilean \\ Ministry of Education, to a University \\ in Medellín. The purpose was to learn \\ about their experience in the initial \\ training of teachers in Colombia, in such \\ a way to contribute with experience \\ and knowledge to the training of future \\ teachers from the Education Faculty \\ of a regional university of the Council
} differences in training pre-service teachers, highlighting the difference between the training of researcher skills in Early Childhood Education students. The analysis permitted arguing about the need to train researcher skills in future teachers to allow the development of pedagogical research in Chile, and thus solve problems tending to improve the quality of education at all levels.

Keywords: Educational research, researcher skills, teacher training.

\title{
Contextualización
}

Durante las últimas décadas, diversos países en América Latina han focalizado la mirada buscando la forma de mejorar el desempeño escolar de los estudiantes y evidenciar logros significativos de sus aprendizajes. Investigaciones como las de Román (2015) evidencian que "la escuela importa y aporta significativamente a lo que aprenden o dejan de aprender los estudiantes” (p.93), por lo que se asume que el profesor tiene una gran responsabilidad, pues lidera el proceso en el aula, lo cual reclama una actualización permanente para dar respuesta a las necesidades e intereses que presenten sus estudiantes. Por este motivo, los gobiernos relevan la formación de profesores en la agenda y su preocupación por el tema, a través del establecimiento de nuevas políticas en la carrera docente, que permiten el reconocimiento explícito a su labor docente, mayor exigencias a las instituciones formadoras de profesores, entre otras.

En el hemisferio contrario a América Latina, Finlandia es reconocido como un país exitoso en cuanto a desempeño escolar y logro académico de sus estudiantes. En ese país se espera que sus profesores se responsabilicen por el aprendizaje y bienestar de sus estudiantes, lo que en opinión de Niemi (2015), "requiere de un alto nivel de competencia pedagógica y un rol profesional amplio" (p.390). El mismo autor señala que las autoridades de ese país han comprendido que es posible formar un profesor con esas características a través de un Desarrollo Profesional Docente (DPD), el que comienza en la Formación Inicial de Profesores (FIP) y continúa hasta su jubilación, lo que entienden como un proceso permanente de actualización durante el ejercicio de la profesión a lo largo del tiempo.

A modo de contextualización histórica, a partir de la década del 90, Chile comienza una nueva etapa en su historia política, reconociendo a la educación como "una estrategia orientada a consolidar el gobierno democrático, promover y apoyar el 
desarrollo económico y social" (Díaz, Solar, Soto, Conejeros \& Vergara, 2015, p.544) y se focaliza en recuperar el rol docente en la sociedad chilena como protagonista de la formación de los ciudadanos del futuro del país. Es así que se responsabiliza como Estado por el DPD, el que al inicio de esta etapa estuvo enfocado más bien en la actualización de conocimientos disciplinares y pedagógicos del profesor en ejercicio. Así también asume responsabilidad para exigir a las universidades la formación de los futuros profesores que el país necesita, y en consonancia con los requerimientos los requerimientos de la sociedad globalizada. En respuesta a estas nuevas exigencias, las universidades comienzan a implementar procesos formativos articulados con las necesidades del mundo laboral y se visualiza a la educación de los ciudadanos como la forma de transformar social y económicamente la sociedad chilena, de tal forma de alcanzar estándares de vida de calidad y más equitativos entre sus miembros.

En esta línea, los esfuerzos se vuelcan hacia el fomento del desarrollo científico y tecnológico que Chile requiere para alcanzar un desarrollo social y económico más avanzado y equitativo. Desde entonces hay un incremento en las inversiones en tecnologías y desarrollo de proyectos de innovación como una forma de aportar a resolver problemas sociales, como también la formación de capital humano calificado para llevar a cabo tal desafío. Para alcanzar ese capital humano, se requiere una formación inicial de los profesionales que los forme en competencias investigativas, por lo que la función investigativa de las universidades adquiere mayor relevancia. Las instituciones de educación superior son las encargadas de desarrollar dichas competencias en los futuros profesionales, como una forma de aportar soluciones en los contextos donde ellos se desenvuelven, ya que hasta ese entonces no ha habido preparación durante su enseñanza superior (Hidalgo y Pérez, 2015). Es aquí donde se observa una incoherencia entre las necesidades de Chile y los montos de inversión económica realizada por el Estado, ya que los profesionales no estaban siendo formados en investigación por las instituciones de Educación Superior durante el pre-grado.

\section{Formación de profesores en investigación}

La FIP fue regulada por el Estado Chileno, principalmente a través de dos documentos ministeriales, el Marco para la Buena Enseñanza (MINEDUC, 2008) y los Estándares Orientadores para la Formación Inicial de Profesores (MINEDUC, 2012), para las distintas disciplinas y niveles educativos. Al realizar una revisión de estos referentes, se observa en ambos la formación en competencias investigativas e innovadoras no queda del todo explícita. El Marco para la Buena Enseñanza sólo presenta un indicador vinculado a estas capacidades dentro del dominio de Responsabilidades Profesionales. Señala que "el profesor reflexiona sistemáticamente sobre su práctica” (Mineduc, 2008, p.11). Los Estándares Orientadores, explicitan dos capacidades que debe demostrar todo egresado de una carrera vinculada a la pedagogía: 
1. “Capacidad creativa, espíritu emprendedor e innovación. El egresado demuestra creatividad al generar nuevas alternativas en las soluciones que se plantean. Realiza proyectos por iniciativa propia, asumiendo los riesgos que esto implica. Responde a los requerimientos, demandas sociales y organizacionales, innovando en los procesos a fin de obtener mejores y mayores resultados”

2. "Conocimientos de análisis cuantitativo que le permitan leer, analizar e interpretar los distintos tipos de datos" (MINEDUC, 2012, p.16).

A su vez, uno de los diez estándares pedagógicos comunes a todas las disciplinas, señala que el egresado "Aprende en forma continua y reflexiona sobre su práctica y su inserción en el sistema educacional” (p. 17). Si bien estos estándares para la FIP declaran qué capacidades debe poseer cualquier profesional de la educación y qué se espera de su desempeño futuro, no hay una intención explícita de formar competencias investigativas en los profesores, la que podría complementar otras capacidades como innovación y reflexión. Por otro lado, las Bases Curriculares para la Educación Parvularia (MINEDUC, 2005) es el referente curricular para la educación de niños de $\circ$ a 6 años y señalan claramente el rol de la Educadora de Párvulos explicitando que se le concibe como "permanente investigadora en acción” (p.14) lo que constituye también una parte fundamental de su quehacer profesional.

Si esto es así, cabe entonces preguntarse ¿cómo espera Chile avanzar en el desarrollo social y económico del país si no existen profesionales formados para investigar y brindar solución a problemáticas del país? ¿Cómo se espera que el profesor reflexione críticamente sobre su desempeño y sea un investigador para la mejora de las prácticas docentes, si no cuenta con las herramientas ni capacidades para ello? ¿Cómo la educadora de Párvulos va a llegar a ser una investigadora en acción si no se formó para ello?

Kingdon (2003) señala que en la agenda pública influyen los participantes visibles como el presidente, sus ministros o el congreso; pero también aportan los actores invisibles del sistema, representados por académicos, especialistas y profesores en aula. Pero para que ello ocurra, esos actores que pueden aportar con sustento empírico, teórico y práctico, primero deben existir. Sabido es que la producción científica en Chile se ha incrementado en los últimos años gracias a los incentivos que entrega el MINEDUC y otras organizaciones para que ello sea así, pero aún persiste una baja producción de conocimiento, sobre todo en las ciencias sociales y específicamente en educación. Lo anterior fundamenta aún más la necesidad de formar estas competencias en los futuros profesores. Para que la política educacional de Chile se decida con fundamentos, debe haber expertos nacionales que contribuyan al debate y al desarrollo de mejores disposiciones.

Bajo la mirada de un paradigma tradicional de enseñanza, la profesión docente se entendió como sinónimo de transferir. Hoy, ejercer la profesión docente desde esa perspectiva no es posible, ya que se exige desarrollo de competencias complejas y 
formación de actitudes y valores también complejos, con la perspectiva de desarrollar capacidades en los estudiantes, aspecto que requiere de profesores que posean esas competencias y sepan cómo desarrollarlas en sus estudiantes. En el ideal de realizar transformaciones profundas en educación, se debe contar con profesionales críticos de la realidad, sensibles a los cambios y mejoras que se requieren. De este modo, aportar desde su propia investigación a la mejora en forma sostenida de la calidad de los aprendizajes y de la enseñanza, impactando incluso en las decisiones que generan políticas públicas en este ámbito.

Por el contrario y nuevamente exponiendo al país que lidera los rankings en educación, Finlandia destaca por fomentar en forma explícita que sus futuros profesores sean profesionales reflexivos, críticos e investigadores (Niemi, 2015). Este autor además señala que la capacidad investigativa es desarrollada en los futuros docentes a través de seminarios y/o proyectos de investigación con el objetivo de "aprender sobre la generación de conocimientos y sobre el pensamiento científico crítico” ( $\mathrm{p}$. 393), buscando que los profesores asuman una actitud investigativa sobre su propia acción docente. Niemi y Nevgi (2014) señalan que la formación de profesores en Finlandia desarrolla principalmente el pensamiento científico e independiente de sus profesores, sumado a la curiosidad intelectual, la alfabetización científica y el cuestionamiento del conocimiento y los fenómenos. La FIP finlandesa, distinta a la chilena, aporta argumentos que pueden explicar en parte la diferencia que se observa en el logro de los aprendizajes de los estudiantes.

En otro contexto latinoamericano, está el caso de Colombia. Camacho, Martínez \& Pedraza (2014) realizan una síntesis de la evolución de las políticas educativas en cuanto a la formación de capacidades investigativas en la formación de profesores. Señalan que ya en la década de los 80 se comienza a generar en Colombia una fuerte vinculación entre la investigación y la docencia. El decreto 080 de ese año, el artículo $4^{\mathrm{O}}$ apuntaba a que la educación superior vinculara la investigación con la docencia, promoviendo un espíritu crítico en el estudiante, que le permita asumir con plena responsabilidad las opciones teóricas y prácticas de su perfeccionamiento personal y del desarrollo social. A su vez, los autores señalan que lenta pero continuamente, las políticas en Educación Superior de ese país fueron afianzando el vínculo investigación-docencia para cooperar al desarrollo educativo nacional, regional, institucional y de las comunidades educativas, debiendo todas las universidades e instituciones universitarias con programas de pregrado y postgrado en Educación "disponer de una infraestructura adecuada para el desarrollo de la investigación educativa y pedagógica, poniendo al menos una línea de investigación por cada uno de los programas académicos que ofrecieran” (p. 101).

De esta forma, con la certeza que la formación en investigación era uno de los criterios principales de calidad educativa, se continuó con la formación de lo que denominaron "docente investigador", hasta llegar a la actualidad donde todo programa de formación de docentes debe tener un componente investigativo y es exigido por la Consejo Nacional de Acreditación de Colombia como uno de los indicadores de excelencia 
para reconocer la calidad de los programas e instituciones. En este sentido, Camacho, Martínez \& Pedraza (2014) destacan "los semilleros de investigación, centros de investigación, líneas de investigación de los programas, desarrollo de proyectos de investigación, entre otros, que fomentan una cultura investigativa y coadyuvan a fomentar el espíritu investigativo y las competencias necesarias para investigar" (p.106).

Así, se ha expuesto brevemente la realidad de tres países y como cada uno de ellos está abordando desde sus políticas, la formación inicial de profesores y los propósitos que en cada uno se vislumbran. Los antecedentes aportan a la comprensión de una problemática que se hace cada vez más evidente, cual es la formación de profesores para una educación de calidad que permita a los ciudadanos del país aportar al desarrollo económico y social de un país.

\section{Formación inicial de profesores en Chile y Colombia}

La pasantía realizada en una universidad colombiana de Medellín, estuvo asociada al proyecto institucional PMI15O1 y vincula al rediseño curricular de las carreras de la Facultad de Educación de una universidad regional, perteneciente al Consejo de Rectores de Universidades Chilenas. Fue programada con el propósito de indagar sobre la formación inicial de profesores en esa institución formadora colombiana, incluyendo la formación de Educadoras de Párvulos. De esta forma, se esperaba recabar experiencias y conocimientos que aporten a la actualización de las nuevas propuestas curriculares de las carreras en la facultad.

Uno de los objetivos de la renovación curricular de este proyecto institucional se relaciona con la actualización de los currículos de las carreras de pedagogía, considerando:
a) los estándares orientadores de la formación inicial docente
b) los estándares de calidad
c) el Sistema de Créditos Transferibles (SCT-Chile)
d) el perfil de los estudiantes de acuerdo a los diversos contextos locales
e) la flexibilidad curricular
f) la capacidad de reflexión sobre la propia práctica
g) los avances en las didácticas disciplinarias
h) la capacidad de autoaprendizaje de los estudiantes.

A su vez, la incorporación a través de los planes de estudios de experiencias prácticas en contextos laborales, que articularan las experiencias previas de los estudiantes con sus propios procesos de construcción profesional y de este modo, contextualizar los procesos curriculares y de enseñanza -aprendizaje en un mundo real.

Es así que a través de distintas reuniones se recabó la información necesaria que permitió reflexionar sobre la forma en que se lleva a cabo la FIP de profesores en la universidad chilena con la institución colombiana, específicamente en la formación 
de competencias investigativas de estos futuros profesionales de la educación infantil, con el propósito de aportar al proceso de renovación curricular; en específico, en la formación de competencias investigativas de los futuros profesionales de la educación infantil, al respecto Correa (2017) "en paralelo a su formación profesional, también lo hace con la investigación; requiriendo de un bagaje conceptual y de experiencia en su campo de conocimiento que lo inserte de manera intencionada a la indagación científica” (p.14).

Uno de los aspectos que llamó la atención, por la diferencia epistemológica entre las instituciones, se relaciona con la formación de habilidades investigativas en la institución colombiana, la que se desarrolla desde el $1^{\mathrm{o}}$ semestre del programa, a través de los cursos de práctica, a la que se incorporan distintos elementos para favorecer el aprendizaje de técnicas de recogida y registro de información. Las primeras prácticas incluyen la observación estructurada y participante, en las intermedias trabajan los estudios de caso, para continuar en la de últimos años con entrevistas. La formación finaliza con la metodología propia de la investigación acción en las prácticas finales. Además, la formación en investigación de los estudiantes se articula con los grupos de investigación, destacando por su coordinación y por la forma como con ello, se beneficia además la docencia, investigación y extensión. Los instrumentos que utilizan para recoger la información, también se encuentran intencionadamente graduados en la medida que avanzan en los distintos cursos de práctica. Paralelo a las prácticas, el itinerario formativo considera cursos de investigación: Investigación-educación, Paradigmas cualitativos-cuantitativos, Métodos de sistematización de prácticas, Experiencias innovadoras en educación infantil.

Por su parte, la organización de la práctica en la universidad colombiana reconoce tres modalidades en que se organiza la Práctica Profesional: institucional, que se traduce en un proyecto pedagógico implementado en aula; emprendimiento, cuyo producto es un proyecto de innovación y emprendimiento e investigación, que considera como producto un artículo de investigación o un capítulo de libro. Esta última modalidad es desarrollada a través de los Seminarios de Investigación, que sumada al Semillero de Investigación del grupo de investigación "Senderos" se diferencia en gran medida del curso de Seminario de Investigación de la universidad chilena. Los Seminarios de Investigación en Colombia corresponden a grupos pequeños de discusión sobre temas referidos a los proyectos de investigación vigentes. Al interior del Seminario, el estudiante realiza lo que se llama protocolo y relatoría.

Las competencias investigativas en las estudiantes de pre-grado marcan una diferencia importante de ambas instituciones. La formación de las Licenciadas en Pre-escolar de la universidad colombiana incorpora en el plan de estudios el desarrollo de competencias investigativas vinculadas con la práctica pedagógica, las que aportan a la implementación de experiencias innovadoras y significativas. El proceso se inicia en cada nivel con la formulación de una pregunta pedagógica o investigativa que los estudiantes deben responder mediante la aplicación de técnicas de investigación (Begué, 2010, p.19-20). Al mismo tiempo, lo hacen utilizando métodos y técnicas de 
investigación que contribuyan a dar significado al saber pedagógico y al objeto de estudio de la profesión. Esto significa desarrollar en las estudiantes competencias para fundamentar epistemológicamente la elección de paradigmas y enfoques de investigación que permitan explorar la evolución del objeto de formación, seleccionar métodos y técnicas de investigación para resolver problemas educativos y pedagógicos y realizar innovaciones; reflexionar sobre las prácticas pedagógicas y su impacto en los sujetos que se están formando; elaborar textos escritos que den cuenta de la resignificación de los objetos de formación; implementar en la práctica pedagógica propuestas, proyectos y estrategias, educativas y pedagógicas, diseñadas en torno al objeto de estudio; implementar sistemas didácticos orientados a la organización de la acción pedagógica, en diferentes contextos y con poblaciones diversas; evaluar el impacto de la interacción e intervención pedagógica, que se evidencia en la gestión de propuestas, proyectos y estrategias, educativas y pedagógicas y socializar las construcciones elaboradas en torno al saber pedagógico.

Por su parte, la universidad chilena instala en los últimos años de la formación de Educadoras de Párvulos, cursos vinculados con el desarrollo de habilidades investigativas, en gran medida es refrendado en el perfil de egreso, en el cual no se hace alusión explicita al desarrollo de estas capacidades ya que señala que "en tanto Licenciado en Educación, el profesional de la Educación Parvularia se compromete con su desarrollo profesional continuo en aspectos disciplinares y pedagógicos, la reflexión sobre sus prácticas, la utilización de las tecnologías y el trabajo con la comunidad educativa en la que se desempeña”. Es así que las competencias investigativas se desarrollan sólo en dos actividades curriculares del plan de formación, las que son Metodología de la Investigación, en el sexto semestre de la carrera y Seminario de Investigación, en el séptimo semestre de un total de ocho. Esta organización curricular sitúa a la investigación en un plano inferior de las competencias específicas que debe desarrollar un estudiante de pre-grado, su incorporación en la malla curricular pareciera ser un requisito de egreso más que una herramienta de trabajo futura.

Por otro lado, para desarrollar la práctica en la modalidad investigación en la universidad colombiana, el estudiante debe haber participado en el grupo Semillero y complementar su línea de formación con la línea de investigación del profesor guía en su grupo. Por lo tanto, la elección se encuentra supedita al mérito que la estudiante ha realizado durante sus años de formación de pre-grado. Las tres modalidades de práctica (intervención, emprendimiento e investigación) permiten a las estudiantes elegir un camino futuro de trabajo, en algunas de las 3 áreas mencionadas, brindando así mayor oportunidad de desarrollo profesional a las estudiantes. En cambio en la universidad chilena la Práctica Profesional está orientada exclusivamente al diseño, implementación y evaluación de proyectos de intervención en aula. La evidencia encontrada en Medellín hace pensar que la formación inicial de las estudiantes de Licenciatura en Pre-escolar en cuanto al desarrollo de habilidades investigativas se encuentra suficientemente desarrollada. Promueve en ellas el gusto por indagar, ven en sus profesores equipo sólidos, con trayectoria y evidencias que respaldan su línea 
y habilidades de investigación, por lo que es posible que las estudiantes perciban el desarrollo de estas competencias como una parte más de su formación, así como de su futuro desempeño docente, asegurando que sea una habilidad incorporada en su práctica docente cotidiana, como la forma que le permita identificar problemáticas educativas y buscar soluciones.

Cabe destacar que las prácticas pedagógicas en la universidad colombiana pertenecen al núcleo de prácticas e investigación, lo que significa una estrecha unión entre ambas áreas. Es decir, la práctica está estrechamente vinculada a la investigación, con el propósito de formar educadores con competencias investigativas sobre su propia acción docente, reflexivos y críticos, que aporten a las mejoras de la educación. En esta institución, la formación en investigación se logra a través de un desarrollo continuo, graduado y diferenciado desde primer año de universidad, siendo incorporada en los cursos de práctica pedagógicas, como en otros cursos de la malla. Busca potenciar las competencias investigativas de los estudiantes, así también, desarrollar una actitud reflexiva y crítica, fomentando el interés por investigar.

\section{Conclusiones}

La formación en investigación observada en la universidad colombiana es parte de los requisitos que deben cumplir todas las instituciones formadoras, acorde a la ley establecida por el Ministerio de Educación Colombiano, por ende, no depende de la voluntad o interés de cada institución. Esto marca una gran diferencia con la realidad de las universidades regionales chilenas, ya que la formación en investigación se remite a la aprobación de un único curso de Metodología de la Investigación y posterior Seminario de Investigación que conduce a una investigación y posterior defensa, luego de la cual las estudiantes obtienen el grado de Licenciatura, independiente del título de Educación de Párvulos. En cambio en la universidad colombiana, el grado de Licenciatura se va formando de manera paralela al título profesional y por ende, está presente durante todo el plan de estudios.

También es destacable la organización curricular de la Facultad de Educación y Ciencias Sociales de la universidad colombiana, ya que favorece la productividad de los docentes adscritos a la Facultad y por ende, fortalece los índices de productividad de la institución. Lo anterior se logra con la vinculación que existe entre práctica e investigación, lo que permite la cooperación y participación de los estudiantes en distintos proyectos y publicaciones de los docentes, a través de los Semilleros de Investigación.

En cambio, la realidad de la universidad chilena en este aspecto es diferente y es altamente probable que sea una situación generalizada en las universidades chilenas, sobre todo las que se ubican fuera de la Región Metropolitana, por ser instituciones de corta data y con acceso a menores recursos económicos provenientes del MINEDUC. Aun cuando, Díaz et al. (2015) concluyen en su estudio que tanto profesores del sistema escolar como universitario chileno, muestran interés por empoderarse de una cultura innovadora, dando 
un gran valor a investigación; al mismo tiempo investigan poco, dadas las condiciones labores adversas en las que se desempeñan, tal como la falta de tiempo para dedicarse a la investigación, tanto a nivel universitario como escolar. La evidencia presentada en ese estudio muestra que, aun cuando la formación preparara a los profesores para llevar a cabo investigación en el aula, no existen condiciones para que ello ocurra, por lo que se hace necesario analizar la forma como las instituciones favorecen u obstaculizan procesos investigativos en sus docentes, tanto a nivel universitario como escolar, ya que la problemática se observa en ambos niveles educativos y evidentemente debe ser mejorada. La investigación que pueda hacer un docente en su aula, puede ser tan significativa como la que puede realizar un docente en el aula universitaria. Ambas contribuyen a la mejora de procesos de enseñanza y aprendizaje, por ende aportan a la calidad de la educación.

Pero lo anterior implica organización de tiempos, de equipos de trabajo, valoración de las propias prácticas en aula, concientización en directores y facultativos de las instituciones, asignación de recursos económicos, ajustes a los requerimientos solicitados a las universidades en cuanto a formación inicial de profesores, ajustes a los estándares orientadores de la formación inicial de profesores para explicitar la formación de habilidades investigativas en los estudiantes de pre-grado. Así también requiere coherencia en quienes toman las decisiones políticas educativas, ya que si se espera alcanzar un desarrollo social y económico acorde con los estándares de los países desarrollados como Finlandia, es necesario favorecer la investigación desde todos los ámbitos para encontrar soluciones a las problemáticas chilenas, especialmente las vinculadas a la educación, base del desarrollo de todo país.

Chile requiere contar con más investigadores que aporten conocimiento acorde al contexto nacional, pero primero estos deben ser formados. La experiencia observada en la universidad colombiana, entrega luces respecto de cómo debiera realizarse la renovación curricular de los planes de estudios de las carreras de la Facultad de Educación de la universidad regional chilena, esto es explicitando el desarrollo de habilidades investigativas, en sus distintos cursos y a lo largo de toda su formación. Así también, debe asegurar su vinculación con las Prácticas Progresivas, las que sí se realizan desde los primeros semestres de estudio.

Resulta necesario insistir que es a través de la educación que los ciudadanos de un país logran desarrollarse y obtienen las herramientas necesarias para aportar al desarrollo del país y aspirar a una buena calidad de vida, y ello se vincula directamente al desarrollo científico y tecnológico del país, de forma de generar soluciones, recursos y conocimiento. Los responsables de dicha formación van desde el gobierno, a través del Ministerio de Educación, hasta cada profesor que forma profesores, de ahí la importancia de formar docentes competentes en todas las áreas y niveles de educación.

Sería interesante indagar qué ocurre en otros países latinoamericanos respecto a la formación de competencias investigativas durante la formación inicial de profesores, qué prioridad se está dando a esta temática desde los gobiernos y desde las instituciones formadoras, cómo las políticas en educación facilitan la formación de estas habilidades 
en los futuros docentes, qué posibilidades reales tienen los docentes del nivel escolar y universitario para realizar investigación en forma paralela a sus labores docentes. Preguntas que sin duda pueden aportar a la discusión.

Finalmente, es importante señalar que la posibilidad de conocer in situ otras realidades de la formación de profesores, permite analizar realidades cercanas. Es a través de la reflexión docente que se puede establecer diferencias y similitudes entre instituciones, para rescatar aquello que puede ser un aporte a la propia realidad, aspecto que se ha logrado con esta pasantía.

\section{Referencias}

Begué, A. (2010). El desarrollo de habilidades investigativas: un eje en el proceso de formación de licenciados en educación. Revista Senderos Pedagógicos (1) 13-22.

Camacho, D., Martínez, C., Pedraza, A. (2014). Políticas educativas y cultura investigativa en la formación de educadores. Revista Educación y Desarrollo Social, 8(2), 96-107.

Correa, J. (2017). Acercándonos a la investigación desde la formación en el pregrado. Revista Funlam Journal of Students Research (2), 13-16.

Díaz, C., Solar, M., Soto, V., Conejeros, M. \& Vergara, J. (2015). Temas clave en la formación de profesores en Chile desde la perspectiva de docentes y directivos. Revista Complutense de Educación, 26(3), 543-569.

Hidalgo, S. \& Pérez, A. (2015). La investigación formativa y su relación con la empresa y el estado como parte del proceso educativo. Zona Próxima, 23, 145158. DOI: http://dx.doi.org/10.14482/zp.22.5832

Kingdon, J. (2003). Agendas, alternatives, and public policies. Addison-Wesley Educational Publishers Inc. U.S.A

MINEDUC, (2005). Bases Curriculares para la Educación Parvularia. Recuperado de http://portales.mineduc.cl/usuarios/convivencia_escolar/ doc/201103041242340.Bases_Curriculares_de_Educacion_Parvularia_2001.pdf

MINEDUC, (2008). Marco para la Buena Enseñanza. Recuperado de http:// portales.mineduc.cl/usuarios/cpeip/File/Documentos\%202011/MBE2008.pdf

MINEDUC, (2012). Estándares Orientadores para Egresados de Carreras de Pedagogía en Educación Básica. Recuperado de http://portales.mineduc.cl/ usuarios/cpeip/File/2012/librobasicaokdos.pdf

Niemi, H., (2015). Desarrollo profesional docente en Finlandia: Hacia un enfoque más holístico. Psychology, Society \& Education, 7(3), 240-251.

Palamidessi, M., Gorostiaga, J., \& Suasnábar, C. (2014). El desarrollo de la investigación educativa y sus vinculaciones con el gobierno de la educación en América Latina. Perfiles educativos, 36(143), 49-66. Recuperado en 05 de octubre de 2017, de http://www.scielo.org.mx/scielo.php?script=sci_ arttext\&pid=So185-26982014000100004\&lng=es\&tlng=es

Román, M., (2015). Investigación latinoamericana sobre enseñanza eficaz. Educación y ciudadanía, 19, 81-95. 